Revue de l'Institut des langues et cultures

d'Europe, Amérique, Afrique, Asie et Australie

43 | 2021

Images des Amériques : fabrique, représentations, usages

\title{
Brésil des Brésiliens (1983), enjeux et stéréotypes d'une exposition destinée à montrer le « Brésil contemporain »
}

Brazil of the Brazilians (1983), Stakes and Stereotypes of an Exhibition Intended to Show "Contemporary Brazil"

Jessica Blanc

\section{OpenEdition}

\section{Journals}

Édition électronique

URL : https://journals.openedition.org/ilcea/13309

DOI : 10.4000/ilcea.13309

ISSN : 2101-0609

Éditeur

UGA Éditions/Université Grenoble Alpes

\section{Édition imprimée}

ISBN : 978-2-37747-299-4

ISSN : $1639-6073$

Référence électronique

Jessica Blanc, «Brésil des Brésiliens (1983), enjeux et stéréotypes d'une exposition destinée à montrer le « Brésil contemporain » », ILCEA [En ligne], 43 | 2021, mis en ligne le 30 juin 2021, consulté le 30 juin 2021. URL : http://journals.openedition.org/ilcea/13309; DOI : https://doi.org/10.4000/ilcea.13309

Ce document a été généré automatiquement le 30 juin 2021.

(C) ILCEA 


\title{
Brésil des Brésiliens (1983), enjeux et stéréotypes d'une exposition destinée à montrer le « Brésil contemporain »
}

\author{
Brazil of the Brazilians (1983), Stakes and Stereotypes of an Exhibition \\ Intended to Show "Contemporary Brazil"
}

Jessica Blanc

1 L'exposition collective Brésil des Brésiliens s'est tenue à la Bibliothèque publique d'information (Bpi), située à l'intérieur du Centre Georges Pompidou à Paris (du 15 juin au 19 septembre 1983). Les cent-douze photographies exposées devaient présenter les différents aspects de la vie au Brésil dans le contexte du début des années 1980. Même si la Bpi avait déjà organisé plusieurs expositions de photographie, il s'agissait ici de la première monographie consacrée à un pays d'Amérique latine ${ }^{1}$. L'objectif, tel que revendiqué à la fois dans le dossier de presse et dans le catalogue de l'exposition, visait à montrer un pays en plein développement, libéré de ses stéréotypes : «Centrée sur la vision du Brésil actuel par les photographes brésiliens, l'exposition s'éloigne à la fois de l'image stéréotypée de l'exotisme, du carnaval, des plages et de celle d'un misérabilisme facile ${ }^{2}$.»

2 Cependant, l'organisation d'expositions au sein même de la Bpi revêtait un enjeu particulier comme l'affirmait le directeur René Fillet ${ }^{3}$ à l'occasion du recrutement de son successeur en 1983 : «Je cherche un successeur. Il faudrait un jeune, qui s'occupe de l'image et de l'audiovisuel, qui fasse de belles expositions, car on nous reproche d'être les parents pauvres, à côté du Musée du Centre Pompidou. Il faut faire mieux dans les animations et dans les nouvelles technologies de l'image.» (Grognet, Danis \& Gaudet, 2006)

3 Dans cette double perspective, d'une part, de proposer un panorama le plus authentique possible du Brésil et, d'autre part, la nécessité d'organiser de belles 
expositions, quelles images sélectionner pour le public de la Bpi ? Les photographies choisies permettaient-elles vraiment de s'éloigner des stéréotypes communément associés au Brésil?

4 La scénographie accordait une place prédominante à la photographie en noir et blanc $^{4}$ et prévoyait un espace consacré aux photographies en couleur, situé à part des sept macrostructures thématiques ${ }^{5}$. D'un point de vue esthétique, la préférence pour le noir et blanc s'inscrit dans la traditionnelle valorisation du reportage d'auteur ${ }^{6}$, souvent privilégiée par les musées et les galeries d'art. La sélection thématique et la scénographie de Brésil des Brésiliens révèlent à plusieurs égards une vision romantique et stéréotypée d'un pays qui était alors encore dirigé par un régime dictatorial militaire.

5 L'une des caractéristiques de cette exposition réside notamment dans le profil des photographes sélectionnés. Tous étaient de nationalité brésilienne, en activité au moment de l'exposition, et beaucoup étaient de jeunes photojournalistes. En quoi cette nouvelle génération de photographes professionnels permettait-elle d'atteindre les objectifs définis pour cette exposition?

\section{Les acteurs du renouveau du photojournalisme au Brésil dans les années 1980}

6 Les textes produits autour de l'exposition, c'est-à-dire le dossier de presse et le catalogue de l'exposition, mettaient en avant les particularités du champ photographique au Brésil dans les années 1980. D’une part, ils évoquaient l'évolution de la photographie brésilienne au cours des cinq dernières années et, d'autre part, ils distinguaient deux grandes tendances au sein de la photographie contemporaine : une préoccupation esthétique et la volonté de fixer la réalité sociale et quotidienne.

7 Ce sont ces récentes évolutions, portées par une génération de jeunes photographes, notamment les photojournalistes indépendants ou regroupés en coopératives, que les organisateurs ont sélectionnés pour l'exposition Brésil des Brésiliens. Comme l'indiquait Roberto Pontual, l'un des auteurs du catalogue d'exposition, ces changements étaient liés à l'évolution de la situation politique du pays (Pontual, 1983:65). Après les «années de plomb» [Anos de Chumbo ${ }^{7}$, une période particulièrement répressive où la censure avait exercé un fort contrôle sur les médias, s'ouvrait alors une période de relative ouverture. À cela, s'ajoutaient dès les années 1970 de multiples facteurs favorisant le développement de la photographie. L'apparition et la multiplication de canaux de diffusion - galeries spécialisées, départements de photographie des musées, expositions, festivals dédiés, presse spécialisée, etc. - et les réformes éditoriales des principaux organes de presse nationaux (Estado de São Paulo, Folha de São Paulo et Jornal do Brasil, par exemple), ont permis aux photographes de développer leur activité et de toucher un plus large public. Animés par un désir de reconnaissance et de professionnalisation de leur métier, les jeunes photographes qui ont débuté leur activité professionnelle au cours des années 1970 et jusqu'au début des années 1980, se sont organisés et regroupés en agences. Ces structures prenaient pour modèle les grandes coopératives internationales telles que l'agence Magnum Photos. 
Comme le précisait le dossier de presse, il s'agissait principalement de jeunes professionnels que les organisateurs avaient sélectionnés : «Le Brésil présenté ici est bien celui des photographes brésiliens dont un grand nombre est presque inconnu en France ${ }^{8}$.» Au total, quarante-quatre photographes de nationalité brésilienne ont été exposés, la moyenne d'âge se situant aux alentours de 39 ans. Parmi les plus jeunes, on peut citer par exemple Maurício Simonetti (24 ans) et Ricardo Malta (27 ans), tous deux appartenant à l'Agência F4. S'il est vrai que la plupart des photographes de l'exposition étaient alors inconnus du public français, cette intention revendiquée officiellement, doit toutefois être nuancée. En effet, Sebastião Salgado était de loin le photographe le plus visible au sein de l'exposition ( 15 photos) ainsi que dans le catalogue (12 photos). Exilé depuis 1969, il vivait à Paris depuis 1974 et avait intégré successivement les agences Sygma (1974-1975), Gamma (1975-1979) puis Magnum Photos (1979-1994), et il avait déjà publié en France l'ouvrage Les Hmongs (Chêne/Hachette, 1982) ${ }^{9}$. De même, Miguel Rio Branco avait intégré Magnum en 1978 (élu associé en 1980) et avait remporté en 1982 le prix Kodak de la critique photographique à Paris ${ }^{10}$.

Outre Magnum Photos, la sélection finale incluait cinq agences brésiliennes: Agil, Agência F4, Jornal do Brasil, Manchete et O Estado. Si Magnum Photos est la seule agence à ne pas être brésilienne, c'est pourtant celle qui fournissait le plus de photographies ( 23 photos exposées), suivie de l'Agência F4 (16 photos exposées). L'Agência F4 (fondée à São Paulo en 1979) et Agil (fondée à Brasilia en 1980) étaient des coopératives de photographes brésiliens indépendants, tandis que Jornal do Brasil, Manchete et $\mathrm{O}$ Estado étaient les agences photo d'organes de presse brésiliens ${ }^{11}$.

D'autres photographes exilés après le coup d'État de 1964 et résidant en France faisaient également partie de la sélection. Ils rassemblaient au total près d'un tiers des œuvres exposées, soit 37 photographies. Leur participation permettait d'insister sur les liens culturels entre la France et le Brésil. En offrant une large visibilité à deux photographes de la célèbre agence Magnum, les organisateurs assuraient ainsi à l'exposition le bénéfice de la notoriété et du prestige de cette agence tout en respectant les objectifs prédéfinis.

11 Pour ces jeunes photographes, l'exposition présentait un réel enjeu en matière de diffusion internationale et l'espoir de nouvelles opportunités de travail. Comme l'a exprimé Joaquim Paiva (1983), il s'agissait d'une certaine reconnaissance de l'existence d'une production photographique brésilienne de qualité : "[L'exposition] atteste de l'importance de notre photographie contemporaine à l'extérieur, déjà reconnue lors des deux colloques de Mexico comme une photographie forte, incisive et critique.» C'est effectivement une période d'internationalisation de la production photographique brésilienne, portée par de jeunes professionnels qui tentent de rivaliser avec les modèles européens et américains. Par exemple, au même moment, la Photographers Gallery de Londres présentait elle aussi les œuvres de jeunes photographes brésiliens ${ }^{12}$ à travers l'exposition collective New Photographers in Brazil (du 20 mai au 18 juin 1983). Stefania Bril - membre externe du comité d'organisation de l'exposition Brésil des Brésiliens et photographe exposée - reconnaissait également un enjeu commercial: "L'exposition devrait ouvrir le marché international des photographes brésiliens, qui sont excellents ${ }^{13}$. » 


\section{Le Brésil, entre objectivité et romantisme exotique}

12 Alors qu'au moment même où se tenait l'exposition le pays était gouverné par un régime dictatorial militaire, certaines photographies sélectionnées abordaient avec audace la thématique politique. À la période la plus répressive, les " années de plomb ", a succédé une vague de contestations et de grèves qui, dès la fin des années 1970 puis, plus encore, au début des années 1980, a contribué à affaiblir la dictature jusqu'à sa chute en 1985. Dans ce contexte, les photographies de l'enterrement de Glauber Rocha ${ }^{14}$, ou d'ouvriers en grève photographiés par Juca Martins ${ }^{15}$, permettaient de donner de la visibilité sur un plan international à celles et ceux qui s'opposaient au régime. Toutefois, les légendes dans le catalogue de l'exposition ne reflètent pas toute la dimension contestataire de ces œuvres et l'on peut se demander si le public parisien était en mesure d'en saisir tout le sens. D'après les documents préparatoires de l'exposition, il ne semblait pas y avoir de textes informatifs à proximité des photographies exposées. Sans plus d'explication, ces deux photographies, par exemple, laissaient à penser qu'il ne s'agissait que d'un enterrement et d'une grève d'ouvriers dépourvus d'une dimension politique contestataire particulière.

Les organisateurs de l'exposition ont pris soin de mettre en garde le public, dans le dossier de presse et dans le catalogue de l'exposition, contre l'exotisme et les stéréotypes. Avant même l'ouverture de l'exposition, ces propos étaient également relayés par la presse ${ }^{16}$. S'il est vrai que certaines photographies remplissaient parfaitement cette mission et permettaient d'offrir au visiteur de la Bpi une documentation contemporaine sur le Brésil, d'autres semblaient s'en écarter et rejoindre les stéréotypes populaires. Les photographies représentant les favelas ou encore le corps de la femme brésilienne permettaient de saisir cette ambiguïté.

14 En première page du dossier de presse, Bernard Bret justifiait le fait de n'avoir sélectionné que des photographes de nationalité brésilienne pour bien manifester la volonté de ne pas tomber dans les stéréotypes habituels qu'il résume ainsi : «de l'exotisme, du carnaval, des plages et de celle d'un misérabilisme facile ${ }^{17}$ ». Si, en toute logique, le regard que portent les photographes brésiliens sur leur propre culture diffère de celui des photographes étrangers, les choix iconographiques faits par les organisateurs de l'exposition sont également à prendre en considération dans l'étude des stéréotypes. En effet, en analysant l'ensemble des 88 photographies publiées dans le catalogue de l'exposition, il est possible de repérer à travers plusieurs œuvres une vision du Brésil teintée de romantisme et d'exotisme hérités de stéréotypes anciens ${ }^{18}$. Cela signifie-t-il que les photographes brésiliens produisaient eux-mêmes des images stéréotypées ou bien la sélection subjective des organisateurs a-t-elle tout simplement contribué à (re)produire une vision stéréotypée du Brésil ?

Concernant la représentation des favelas par exemple, seules trois photographies abordent cette thématique. Dans la photographie en noir et blanc de Carlos Freire, une jeune femme tient dans ses bras un nouveau-né tandis que l'on distingue en arrièreplan les habitations précaires de la favela Morro Azul à São Paulo. Le visage serein et légèrement souriant, l'attitude protectrice envers le nourrisson qu'elle tient dans ses bras laissent à penser qu'il s'agit d'une jeune mère heureuse et bienveillante. Cette réinterprétation contemporaine de la "vierge à l'enfant » offre ainsi une vision douce et romantique du quotidien d'une jeune mère de famille dans une favela du Brésil. Bien que l'architecture chaotique, typique des favelas, permette d'identifier le contexte 
de la prise de vue, aucun autre indice ne renseigne sur la précarité du quotidien dans une zone urbaine particulièrement défavorisée. En revanche, la sélection d'une représentation sereine et positive de la maternité dans le contexte de la favela permet aux organisateurs de parfaitement répondre à leur objectif d'exclure tout événement sensationnaliste.

La photographie en couleur de Juca Martins présente quant à elle une femme en train de laver du linge dans une favela de la ville de Curitiba (État du Paraná). Comme le précise l'auteur, cette photographie a été réalisée pour alimenter la banque d'images que l'agence proposait à ses clients: "Cette photo de favela à Curitiba a été faite en marge d'un travail que je suis allé faire sur la ville pour la banque d'images au début de l'agence $\mathrm{F} 4^{19}$. " La présence féminine est centrée au premier plan, dans la partie basse de la composition. Le torse et la tête de la femme paraissent petits, presque perdus, au milieu du linge suspendu qui occupe les trois-quarts de la composition. L'arrière-plan flou permet de mieux concentrer le regard du spectateur vers les tâches colorées que forment les vêtements suspendus au vent. Si l'activité - laver du linge à la main - évoque un quotidien plutôt laborieux, la composition met plus en avant les lignes anarchiques des fils à linge et les couleurs que l'action en train de se produire. Là encore, la « vision du Brésil actuel », pour reprendre l'objectif énoncé dans le dossier de presse de l'exposition, est une vision paisible d'un geste banal.

Enfin, la photographie d'Hugo Denizart est encore plus surprenante dans la vision qu'elle offre du quotidien dans une favela brésilienne des années 1980. Celle-ci représente un groupe de jeunes garçons nus, sautant dans l'eau depuis une berge bordée de végétation. Les enfants semblent jouer dans une sorte de jardin d'Éden atemporel et isolé du monde puisqu'aucun repère (géologique, architectural ou chronologique) ne permet de localiser la scène. L'intense luminosité naturelle fait scintiller les gouttelettes d'eau en suspension, la végétation et les corps mouillés des enfants. Cet effet, dont le rendu rappelle l'esthétique du pointillisme, contribue à donner une vision esthétisée qui semble très éloignée de la vie quotidienne dans la favela de la Cidade de Deus [Cité de Dieu]. Construite dans la périphérie ouest de Rio de Janeiro à partir de 1964, elle était réputée pour être l'une des favelas les plus violentes jusqu'à la fin des années 1980 . C'est précisément la violence et la précarité qui touchaient les enfants de cette favela qui ont été retranscrits en 2002 dans le célèbre long-métrage Cidade de Deus du réalisateur brésilien Fernando Meirelles. Ce film offre une vision totalement opposée à celle contenue dans la photographie d'Hugo Denizart.

Si ces trois photographies montraient bien des scènes se déroulant dans des favelas brésiliennes au début des années 1980, elles offraient néanmoins une vision tronquée de la réalité. En voulant éviter à tout prix le sensationnalisme et le misérabilisme, la sélection iconographique effectuée par les organisateurs de l'exposition avait ainsi volontairement écarté les problèmes auxquels les habitants des favelas étaient et restent confrontés au quotidien (insécurité, trafics de drogue et d'armes, insalubrité, etc.). Ainsi, ces trois photographies permettaient-elles sans aucun doute de tenir éloignée "la veine facile de "l'esthétique de la misère" (Bril, 1983:52) que les organisateurs critiquaient explicitement, mais n'offraient au visiteur de la Bpi qu'une vision très édulcorée de la vie quotidienne dans une favela.

19 La sensualité des corps, et notamment celle de la femme brésilienne, fait partie des stéréotypes fréquemment associés au Brésil. Pour le regard européen, cette femme exotique exhibe bien souvent ses formes généreuses dans des vêtements minimalistes. 
Plusieurs photographies, exposées et publiées dans le catalogue offrent ainsi une vision érotisée du corps des Brésiliennes. Qu'il s'agisse de femmes dénudées pendant le carnaval, d'un gros plan sur de jeunes fesses en bikini ou bien d'une supportrice de foot lors de la coupe du monde de football de 1982, ce sont dans tous ces exemples les courbes du corps féminin et la charge sexuelle qu'il contient qui sont avant tout mises en avant. L'arrière-plan n'est qu'une masse informe de supporters dans la photo de Samuel Iavelberg ${ }^{20}$ ou un aplat noir dans la photographie de Miguel Rio Branco ${ }^{21}$. Ainsi, les repères spatiaux et l'action qui est en train de se dérouler sont minimes par rapport aux courbes du corps qui se détachent au premier plan et captent l'attention du spectateur. Tout ceci est encore plus évident dans la photographie d'Isabel Garcia ${ }^{22}$. Les fesses en gros plan de jeunes Brésiliennes sur une plage de Rio de Janeiro emplissent le cadrage. Ces femmes n'ont plus ni tête ni jambe, elles se résument à cette unique partie de leur corps tandis que le paysage hétérotopique de la plage carioca n'est pas identifiable puisqu'il se résume à quelques aplats de couleur beige. Dans le catalogue imprimé de l'exposition, cette photographie a été placée en regard de la photographie de Miguel Rio Branco dont la composition est saturée de capots de voitures sur un parking de São Paolo. Dans une sorte de comparaison formelle, la femme-objet brésilienne semble s'entasser sur la plage et saturer la photographie de ses courbes généreuses tout comme les voitures qui envahissent le paysage urbain de la capitale.

Selon Jean Davallon, toute exposition «qui entend faire comprendre quelque chose, de quelque nature que ce soit, au visiteur et qui, pour ce faire, entend lui donner des éléments d'information » (2010: 235) possède une dimension documentaire. Ainsi, les choix qui s'opèrent quant à la sélection des photographes, de leurs œuvres et de la scénographie, servent en premier lieu l'objectif principal d'un projet d'exposition. Un transfert semble ainsi s'opérer, de la valeur documentaire des photographies définie par le photographe vers la dimension documentaire de l'exposition définie par les organisateurs.

21 La juxtaposition d'une iconographie présentant la population brésilienne dans une approche optimiste n'est pas sans rappeler l'exposition The Family of Man. Organisée en 1955 par Edward Steichen pour le MoMA ${ }^{23}$, celle-ci rassemblait 273 photographes du monde entier, professionnels et amateurs, afin de montrer les différences culturelles mais surtout les similitudes entre les peuples. Dans le contexte de la Guerre froide, ces photographies humanistes mettaient en avant tout le côté positif de l'humanité. En 1983, la population brésilienne vivait les dernières années du régime dictatorial militaire. Il s'agissait de la période la plus contestataire, où de grands mouvements de grèves et des manifestations populaires réclamaient le retour à la démocratie. Pour montrer aux visiteurs de la Bpi le Brésil contemporain, les organisateurs ont sélectionné des œuvres pacifistes, positives et dénuées de scènes choquantes. Le quotidien violent et difficile des habitants des favelas, les enfants des rues, les opposants au régime arrêtés et torturés, les populations marginales s'entassant dans les périphéries des grandes villes, par exemple, étaient exclus de la "vision du Brésil actuel ${ }^{24}$ " proposée par l'exposition Brésil des Brésiliens.

Certaines photographies sélectionnées renvoyaient ainsi directement à la volonté d'organiser de «belles expositions » en proposant aux visiteurs une vision poétique contemporaine du Brésil. C'est particulièrement le cas, par exemple, de la photographie de Maureen Bisilliat représentant une jeune ramasseuse de crabes ${ }^{25}$. Cette photographie était issue de la série intitulée As caranguejeiras, réalisée en 1968 dans 
l'État de Paraíba (Brésil). La photographe s'est inspirée du poème écrit en 1949 par João Cabral de Melo Neto, Um Cão sem Plumas ["Un chien sans plumes»] dénonçant les conditions de vie précaires des habitants du Nordeste. Maureen Bisilliat proposait dans cette série photographique une interprétation personnelle d'une tradition locale - la pêche aux crabes - à travers une recherche esthétique qui l'emporte sur la valeur documentaire. L'utilisation du noir et blanc permet de brouiller les limites, les corps et la boue se mélangent pour devenir une sorte de masse informe. Pourquoi choisir en 1983 une telle photographie si l'on souhaitait réellement montrer un "Brésil actuel » au public européen ? Cette photographie semble plutôt illustrer un intérêt pour des photographies dont les qualités esthétiques permettaient de séduire les visiteurs de la Bpi.

Ce sont probablement ces quelques stéréotypes sur le Brésil et la sélection iconographique répondant au double objectif de montrer la réalité contemporaine et en même temps de réaliser une belle exposition, que le collectionneur et photographe brésilien Joaquim Paiva (1983) dénonçait dans sa critique de l'exposition: «C'est un Brésil sans folklore et exotisme, comme l'a revendiqué le Centre [Pompidou], mais un Brésil vu depuis une France hautaine, logique, propre, blanche, supérieure et belle, tout le contraire du "Tiers Monde". Le "Tristes Tropiques" ?»

\section{BIBLIOGRAPHIE}

ALBIGÈs Luce-Marie \& BRIL Stefania (1983), Brésil des Brésiliens, cat. expo. (Paris, Bibliothèque publique d'information, 15 juin-19 septembre 1983), Paris : Centre Pompidou et Bibliothèque publique d'information.

BRET Bernard (1983), Dossier de presse de l'exposition Brésil des Brésiliens, Paris : Bpi \& Centre Georges Pompidou.

BRIL Stefania (1983), « Impressions photographiques », L.-M. Albigès \& S. Bril, Brésil des Brésiliens, cat. expo. (Paris, Bibliothèque publique d'information, 15 juin-19 septembre 1983), Paris : Centre Pompidou \& Bibliothèque publique d'information, p. 52.

CAUjolle Christian (1993), Sebastião Salgado, Paris : Centre national de la photographie.

DAVALLON Jean (2010), «L'écriture de l'exposition : expographie, muséographie, scénographie », Culture \& Musées, 16(1), 229-238.

GRognet Thierry, DANIS Sophie \& GAUDET Françoise (2006), « Entretien avec Michel Melot, directeur de la Bibliothèque publique d'information, de 1983 à 1989 », Bibliothèque publique d'information / Centre Pompidou, en ligne sur DocPlayer : <HTTP://DOCPLAYER.FR/15938162ENTRETIEN-AVEC-MICHEL-MELOT-DIRECTEUR-DE-LA-BIBLIOTHEQUE-PUBLIQUE-DINFORMATION-DE-1983-A-1989.HTML> (10 février 2021).

LAVOIE Vincent (2010), Photojournalismes : revoir les canons de l'image de presse, Vanves : Hazan.

« Miguel Rio Branco » (sans date), en ligne sur Magnum Photos : <https://

pro.magnumphotos.com/C.aspx?VP3=CMS3\&VF[...]> (23 mai 2021).

ILCEA, 43 | 2021 
PAIVA Joaquim (1983), « Brasil dos brasileiros », Fotoreporter, 1(2), Porto Alegre. Archives de Joaquim Paiva.

«Para francês ver, O Brasil dos brasileiros », Visão, 30 mai 1983, p. 88.

PonTUAL Roberto (1983), « Le Brésil dans la photo brésilienne », CNAC magazine, 15.

\section{NOTES}

1. Ce n'est qu'en 1992 que deux expositions temporaires ont été à nouveau consacrées à l'Amérique latine au Centre Pompidou: Art d'Amérique latine 1991-1968 dans la grande galerie ( 5 étage) et Amériques latines, l'univers de Borges dans la galerie nord (mezzanine).

2. Dossier de presse de l'exposition Brésil des Brésiliens, archives de la Bpi, Centre Georges Pompidou, 1983, p. 1.

3. René Fillet a été le second directeur de la Bpi, du 30 août 1977 au 30 août 1983.

4. L'exposition présentait 86 photographies en noir et blanc et 26 en couleur, dans des formats variés.

5. Liste des macrostructures telles qu'elles apparaissent sur le plan de l'exposition conservé par les archives du Centre Pompidou: Paysages; Visages ; Le quotidien; Spiritualité et rites; Vie rurale ; En ville ; Rêve et réalité ; Couleurs.

6. À ce sujet, voir Lavoie (2010: 190-197).

7. Les «années de plomb» s'étendent de la rédaction de l'AI-5 [Acte institutionnel $\mathrm{n}^{\circ}$ 5] le 13 décembre 1968 à la fin du gouvernement d'Emílio Garrastazu Médici en mars 1974.

8. Dossier de presse de l'exposition Brésil des Brésiliens, op. cit., p. 1.

9. Cf. Caujolle (1993).

10. Cf. "Miguel Rio Branco" (sans date), en ligne sur Magnum Photos: <https:// pro.magnumphotos.com/C.aspx?VP3=CMS3\&VF[...]> (23 mai 2021).

11. Jornal do Brasil et $O$ Estado de S. Paulo sont des quotidiens tandis que Manchete était une revue hebdomadaire.

12. Photographes exposés : Orlando Brito, Mario Cravo Neto, Odilon de Araujo, Claudio Edinger, Carlos Freire, Paulo Friedman, Cristiano Mascaro, Gil Prates, João Urban.

13. Cf. « Para francês ver, O Brasil dos brasileiros », Visão, 30 mai 1983, p. 88.

14. Cf. Carlos FreIRE (1983), «Funérailles de Glauber Rocha », dans Brésil des Brésiliens, cat. expo., op. cit., p. 29. Cinéaste, acteur et écrivain brésilien, Glauber Rocha (1939-1981) était un opposant au régime dictatorial notamment à travers ses œuvres. Comme l'ont révélé en 2014 des documents divulgués par la Comissão da Verdade [Commission de la Vérité], le gouvernement militaire le considérait comme un individu dangereux et avait projeté de le tuer. Ce projet d'assassinat n'a pu être réalisé puisque Rocha est décédé en 1981 d'une infection pulmonaire. Voir Tiago DiAs (2014) «Comissão da Verdade revela que militares queriam matar Glauber Rocha ", UOL, <https://cinema.uol.com.br/noticias/redacao/2014/08/16/comissao-da-verdaderevela-que-militares-queriam-matar-glauber-rocha.htm> (14 février 2017).

15. Cf. Juca MARTins (1983), «São Bernardo, rassemblement de métallurgistes », dans Brésil des Brésiliens, cat. expo., op. cit., p. 74.

16. Cf. « Para francês ver, O Brasil dos brasileiros », op. cit.

17. Dossier de presse de l'exposition Brésil des Brésiliens, op. cit., p. 1.

18. C'est le cas par exemple de l'iconographie de la femme noire allaitant son enfant sur un marché visible dans la photographie de Mario CRAVO NETO (1983), «Bahia, mère », dans Brésil des Brésiliens, cat. expo., op. cit., p. 56. 
19. «Essa foto de favela em Curitiba foi feita a parte de um trabalho que fui fazer sobre a cidade para o arquivo no início da Agencia F4.» (Courrier électronique de Juca Martins à Jessica Blanc, 4 avril 2017)

20. Cf. Samuel Iavelberg (1983), «Coupe du monde 1982 », dans Brésil des Brésiliens, cat. expo., op. cit., p. 60.

21. Cf. Miguel Rio Branco (1983), «Rio, carnaval », dans Brésil des Brésiliens, cat. expo., op. cit., p. 83.

22. Cf. Isabel GARCIA (1983), « Rio de Janeiro », dans Brésil des Brésiliens, cat. expo., op. cit., p. 62.

23. L'exposition est à présent visible au musée de Clairvaux, Grand-Duché du Luxembourg.

24. Dossier de presse de l'exposition Brésil des Brésiliens, op. cit., p. 1.

25. Cf. Maureen Bisilliat (1983), "Pêche au crabe », dans Brésil des Brésiliens, cat. expo., op. cit., p. 49.

\section{RÉSUMÉS}

L'exposition collective Brésil des Brésiliens (Bibliothèque publique d'information du Centre Georges Pompidou, Paris, 1983) devait présenter une vision actuelle du Brésil à travers la sélection d'une centaine de photographies. Il s'agissait d'une exposition temporaire destinée à un public français et international curieux de découvrir les réalités économiques, sociales et culturelles d'un pays qui était en train de vivre les dernières heures d'un régime dictatorial. Cet article propose de revenir sur le profil original des photographes sélectionnés puis, sur quelques œuvres permettant de mettre en relief les tensions entre les objectifs définis par les organisateurs. Tout d'abord, l'étude de la sélection des photographes reflète la présence de la nouvelle génération de jeunes Brésiliens sur la scène internationale, notamment ceux qui travaillaient au sein des toutes nouvelles agences de photographie à l'instar de l'Agência F4, par exemple. Puis, l'analyse des œuvres abordant l'environnement urbain des favelas ou bien le corps de la femme brésilienne permet de mettre en évidence des critères sélectifs parfois basés sur l'esthétisme et l'exotisme. En dépit de l'objectif revendiqué par les organisateurs de tenir l'exotisme et le sensationnel à distance, la nécessité de produire de « belles expositions » était également présente.

The group exhibition Brésil des Brésiliens (Bibliothèque publique d'information du Centre Georges Pompidou, Paris, 1983) was to present a current vision of Brazil through the selection of about a hundred photographs. It was a temporary exhibition intended for a French and international public curious to discover the economic, social and cultural realities of a country which was going through the last hours of a dictatorial regime. This article proposes to study the original profile of the selected photographers then, to analyze some works highlighting the tensions between the objectives defined by the organizers. First, the study of the selected photographers reflects the presence of the new generation of young Brazilians on the international scene, in particular those who worked in the very new photographic agencies like Agência F4, for example. Then, the analysis of pictures dealing with the urban environment of the favelas or even the body of Brazilian women allows us to highlight selective criteria sometimes based on aestheticism and exoticism. Despite the goal claimed by the organizers of keeping the exotic and the sensational at bay, the need to produce "beautiful exhibitions" was also present. 
INDEX

Mots-clés : Brésil, exposition, Bibliothèque publique d'information (Bpi), Agência F4, histoire de la photographie, Juca Martins, Miguel Rio Branco, Sebastião Salgado, stéréotype

Keywords : Brasil, exhibition, Bibliothèque publique d'information (Bpi), Agência F4, history of photography, Juca Martins, Miguel Rio Branco, Sebastião Salgado, stereotype

\section{AUTEUR}

JESSICA BLANC

Université Paris 1 Panthéon-Sorbonne

jessica.blanc28@gmail.com 